\title{
Application of Finger Assisted Laparoscopic Surgery (FALs) in Removal of Complex Common Bile Duct Stones
}

\author{
Uma Shanker S, DNB., MRCS. , Sai Srinivas, M.S. , Viswanathan M, Mch. , Swathanthra N. \\ Department of General and Minimally Invasive surgery, Lalitha Super Specialties Hospital, Guntur, Andhra Pradesh, India
}

\begin{abstract}
Objectives: To describe a new surgical technique for the management of complex common bile duct stone and its feasibility along with reproducibility. Methods: We developed a new surgical technique to remove the impacted stone in common bile duct which was previously attempted but failed by one or more ERCP. We did this technique by inserting one finger through the laparoscopic port to negotiate the impacted stone in CBD after failed $L C B D E$ with presently available stone extraction methods and ultimately extracted the large stone from CBD. Results: We did this new technique on three patients who had impacted stone with one or more failed attempts by ERCP and failed by even LCBDE. We succeeded in extracting the stone with this method in all cases without any complication. The time duration to extract the stone by this method are about 5 to 7 minutes only. By adopting this method to extract the impacted large stone in CBD after failed LCBDE, we avoided conversion to open and evaded using sophisticated laser lithotripsy which might cost more with morbidity. Conclusion: The result of the study indicatesthat all the cases with complex CBD stone where clearance is not possible by ERCP/ LCDBE can be removed very easily by this technique without opting for electrohydraulic or laser lithotripsy which consumes more time and cost.
\end{abstract}

Keywords: Complex CBD stone, Finger assisted laparoscopic procedure, Novel technique for CBD stone removal

\section{Introduction}

Laparoscopic surgery has lot of advantages over the conventional surgery. But, it has its own disadvantages like absence of tactile sensation and not always possible to perform complicated procedures. We had a situations where we struggled in doing CBD stone extraction through laparoscopically. We innovatively thought of using fingers (Finger assisted laparoscopic surgery ${ }^{2}$-FALS) through one of the ports to dislodge the impacted stone in CBD and extracted it laparoscopically without conversion. From April 2015 to December 2019 we had 15 cases of complex CBD stone $^{14}$ which was a failed cases of stone retrieval. Initially, we posted all these cases for LCBDE and proceeded. From April 2015 to June 2017 we had 8 cases and later part we did 7 cases with similar situation. Out of initial 8 cases we succeeded with LCBDE only on 3 cases with lot of struggle and consumption of time. Rest of the 5 cases were opened and stone in the CBD was removed. Later on, fromthe ninth case we thought of inserting finger(s) through one of the nearestport to CBD into the abdominal cavity and through tactile sensation we retrieved the impacted stone. We continued the same technique for next LCBDE failed 3 cases out of 7 and succeeded in removing stone from CBD.

\section{Operative Technique}

The procedure starts under general anaesthesia with patient in supine position placed on $\mathrm{C}$ - arm compatible table with head elevated position (reverse trendelenberg position). Through $1 \mathrm{~cm}$ umbilical incision, veress needle inserted into the abdominal cavity and pneumoperitoneum was created targeting the intraabdominal from $12 \mathrm{mmHg}$ to $14 \mathrm{mmHg}$. Once the abdomen is distended with $\mathrm{CO}_{2}$ gas, $10 \mathrm{~mm}$ optical trocar was inserted through the umbilical wound and $30^{\circ}$ scope introduced into the abdominal cavity for primary surveillance. Then, $10 \mathrm{~mm}$ working port was inserted in the midline of abdomen in the epigastric region at the level of liver margin. One $5 \mathrm{~mm}$ port are inserted at the level of fundus of gallbladder along the midclavicular line and another $5 \mathrm{~mm}$ port along the anterior axillary line just below the right subcostal margin( position of working ports may vary according to patients abdominal contour and position of gallbladder). Surgeon will stand on left side of the patient, cameraman will stand on left side of the surgeon and assistant will stand on right side of the patient to retract the gallbladder. Using the Maryland forceps and bipolar forceps Calot's triangle was identified, dissected and cystic artery and duct were identified. Cystic artery was clipped and transected and cystic duct was dissected until to see the dilated Common bile duct. Gallbladder was dissected from the liver bed and cystic duct opened

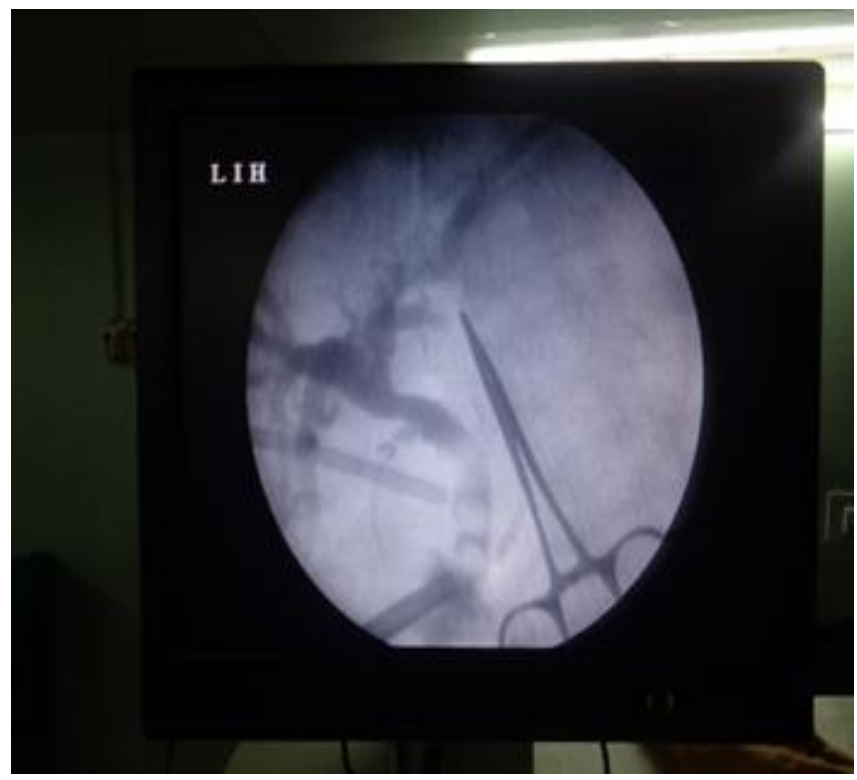

Figure 1: Intraoperative cholangiogram 


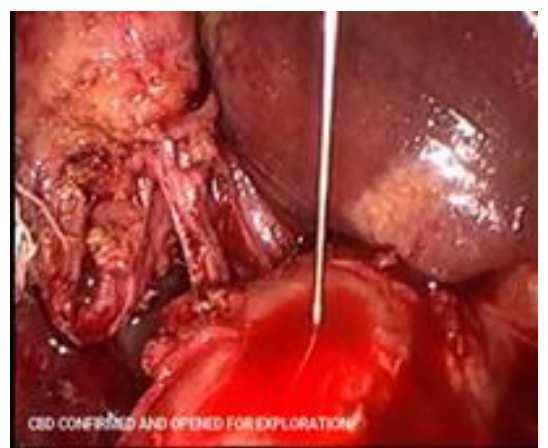

Figure 2: Needle aspiration from $\mathrm{CBD}^{1}$

partially to do intraoperative cholangiogram by inserting the cannula into it (figure -1). Common bile duct was identified by inserting a needle and aspirate for bile(figure -2). Once CBD was identified it was opened vertically with the help of endoknife and looked for bile to flow into the peritoneal cavity. All the debris and bile was sucked out(figure -3) and measures to extract the stone impacted in the CBD are attempted using balloon retraction(figure -4) method or basket retrieval under Choledochoscope

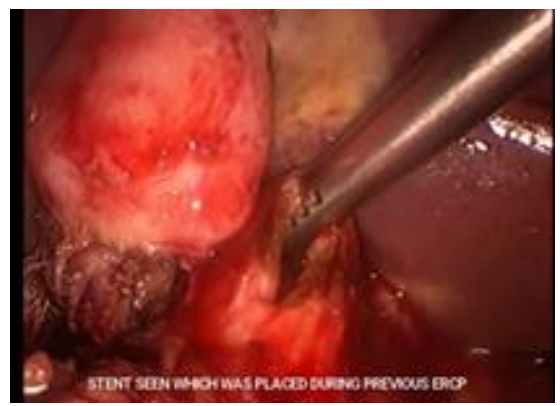

Figure 3: CBD opened and debris sucked ${ }^{11}$

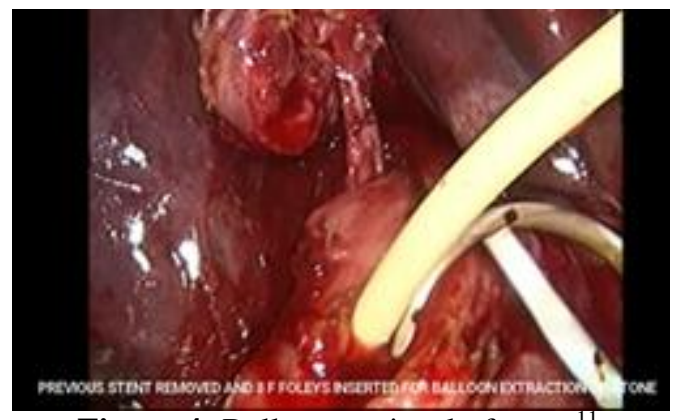

Figure 4: Balloon retrieval of stone

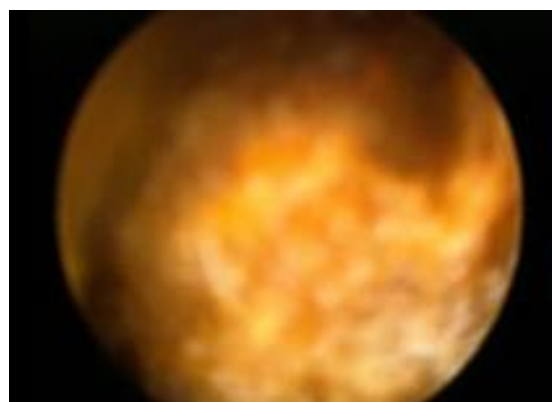

Figure 5: Choledochoscope view of CBD stone ${ }^{11}$

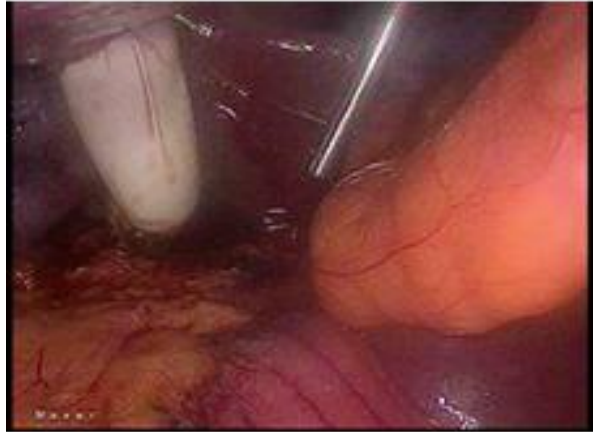

Figure 6: Finger inserted into the abdominal cavity ${ }^{11}$

(Figure 5) etc. Once all the available method are used unsuccessfully we decided to go for insertion of finger (index finger of left hand of surgeon) into the one of the medial subcostal $5 \mathrm{~mm}$ port by making it into $15 \mathrm{~mm}$ (figure6 ). While doing negotiation with stone the intra-abdominal pressure was

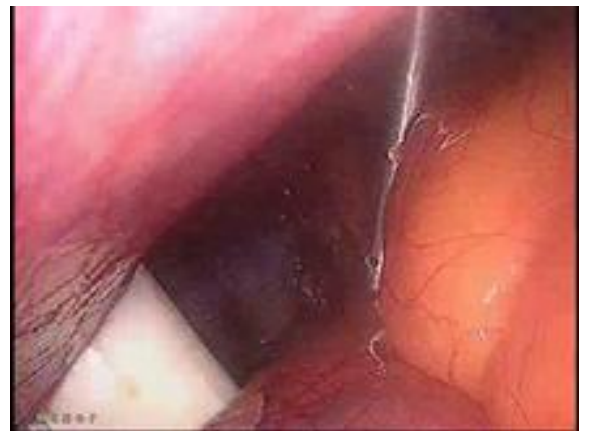

Figure 7: Finger disimpacting the CBD stone ${ }^{11}$

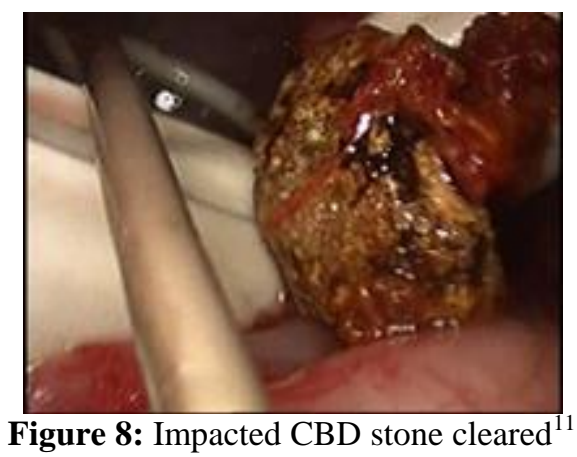

Reduced to $8-10 \mathrm{mmHg}$ so that the surgeon's finger can reach the target comfortably but without compromising the visibility through scope (figure -7). With tactile sensation along with direct vision we could palpate the stone and disimpact it and push it toward cranially until it is visible through the opening made on the CBD (figure -8 ). Once the stone is visible, finger was removed and $15 \mathrm{~mm}$ trocar was inserted into the wound or $5 \mathrm{~mm}$ post inserted and the wound closed with suture to avoid leakage of gas from inside the abdominal cavity. All the extracted stones and removed gallbladder are kept in the retrieval bag and parked somewhere in the abdominal cavity to remove it safely at the end of procedure. Choledochoscope was inserted in to the CBD for the presence of any residual stones. Once CBD stone clearance was confirmed $12 \mathrm{Fr}$ T-tube was inserted into the CBD and the long end was brought out through the closet anterior abdominal wall and secured well. Common bile duct defect are closed with 3-0 polyglactin and tube drain was kept in the Morrison's pouch and brought through 
right flank. Specimens were removed finally from one of the port under vision and send for histopathological examination. All the trocars are removed under vision and wounds are closed with skin staplers after fascial closure with 2-0 polyglactin.

\section{Results}

From April 2015 to December 2019 we posted 15 cases of complex Common bile duct stone ${ }^{14}(\mathrm{CBD})$ for Laparoscopic Common bile duct exploration(LCBDE). Out of initial 8 cases CBD stone clearance was completed successfully on 3 cases only with lot of difficulties and consumption of time and the remaining 5 cases were converted to open surgery and stones are cleared( Table -1). During performing the ninth case we had a similar problem of not able to retrieve stone through any of the available methods and we thought of doing finger assistant for the retrieval of impacted stone in CBD. It took around 7 minutes to disimpact the stone with this method. We adopted this method for the next two cases presented with the same scenario and succeeded in clearance of stone from CBD and avoided conversion to open thus improved the quality of procedure, reduced considerable operative time and surgery related morbidity. In our small study group majority of them are females and with deranged liver function but fit for general anesthesia.From our observation it was found that for the above surgical scenario instead of converting to open or doing electrohydraulic or laser lithotripsy to clear the stone in CBD this novel technique(FALS - Finger Assisted Laparoscopic surgery) may help a lot and reduces the operative time, avoids the morbidity of open surgery and cut down the cost that will be spend of further sophisticated procedures.

Table 1: Case details of complex CBD stone posted for LCBDE from April 2015 to December 2019

\begin{tabular}{|c|c|c|c|c|c|c|c|c|}
\hline Cases & Sex & Age & S. bilirubin & USG report & ERCP attempt & LCBDE result & Open CBD Exploration & FALS done \\
\hline 1 & $\mathrm{~F}$ & $32 \mathrm{y}$ & Normal & Cbd stone & 1 failed & Pass & - & - \\
\hline 2 & $\mathrm{~F}$ & $48 y$ & $3 \mathrm{gm} / \mathrm{dl}$ & Multiple cbd stones & 1 failed & failed & yes & - \\
\hline 3 & $\mathrm{~F}$ & $42 y$ & $4.3 \mathrm{gm} / \mathrm{dl}$ & Impacted 1 cbd stone & 2 failed & failed & yes & - \\
\hline 4 & $\mathrm{~F}$ & $37 y$ & Normal & Cbd stone & 1 failed & failed & yes & - \\
\hline 5 & $\mathrm{M}$ & $53 y$ & $2.5 \mathrm{gmldl}$ & Distal cbd stone & 1 failed & pass & - & - \\
\hline 6 & $\mathrm{~F}$ & $27 y$ & normal & Cbd stone & 1 failed & pass & - & - \\
\hline 7 & $\mathrm{~F}$ & $36 y$ & normal & Cbdstone & 1 failed & failed & yes & - \\
\hline 8 & $\mathrm{M}$ & $32 y$ & $6 \mathrm{gm} / \mathrm{dl}$ & Impacted cbd stone & 2 failed & failed & yes & - \\
\hline 9 & $\mathrm{~F}$ & $47 y$ & $3.4 \mathrm{gm} / \mathrm{dl}$ & Cbd stone & 1 failed & failed & - & yes \\
\hline 10 & M & $35 y$ & $4 \mathrm{gm} / \mathrm{dl}$ & Large cbd stone & 2 failed & failed & - & yes \\
\hline 11 & $\mathrm{~F}$ & $61 y$ & normal & Cbd stone & 1 failed & pass & - & - \\
\hline 12 & $\mathrm{~F}$ & $58 y$ & normal & Cbd stone & 1 failed & pass & - & - \\
\hline 13 & $\mathrm{M}$ & $33 y$ & $2.7 \mathrm{gm} / \mathrm{dl}$ & Multiple cbd stones & 2 failed & failed & & yes \\
\hline 14 & $\mathrm{~F}$ & $45 y$ & $1.5 \mathrm{gm} / \mathrm{dl}$ & Distal cbd stone & 1 failed & pass & - & - \\
\hline 15 & $\mathrm{~F}$ & $39 y$ & $3 \mathrm{gm} / \mathrm{dl}$ & Cbd stone & 1 failed & pass & - & - \\
\hline
\end{tabular}

\section{Discussion}

Common bile duct stones are usually managed by two stage procedure (ERCP followed by Laparoscopic cholecystectomy-LC) or single stage procedure (Laparoscopic cholecystectomy with laparoscopic common bile duct exploration-LCBDE). Patients with failed ERCP for CBD stone is considered as high complex cases ${ }^{9}$. One of the reason for the failure of ERCP in extracting CBD stone is impacted large stone $>1 \mathrm{~cm}^{14}$. In this situation, the options available are LC plus LCBDE or open cholecystectomy with CBD exploration. But, the success rate for laparoscopic common bile duct exploration (LCBDE) after failed ERCP are around $66 \%$ and conversion rates are high ${ }^{9}$. Even though the success rate of CBD stone clearance rates are over $90 \%$ with cholangioscopicelectrohydraulic lithotripsy $(\mathrm{EHL})^{10}$ or laser lithotripsy ${ }^{12}$, the cost and time consumption to complete the task are more. Moreover it may require multiple endoscopic attempts to clear the stone completely ${ }^{13}$. In case of failed LCBDE instead of opting for open or endoscopic lithotripsy, using surgeons finger(s) for dislodgement of impacted stone in CBD which was inserted through one or more of the port site will aid in retrieval of stone along with the help of blunt laparoscopic instruments. We there by achieved back the tactile sensation which is lacking in laparoscopic procedure and prevented vindicated open surgery. More over the time consumed to execute the procedure (FALS) after decided to do so is hardly 5 to 7 minutes from our study.

Finger-assisted laparoscopic surgery (FALS) is affordable and reproducible to perform the advanced laparoscopic procedures. This new technique made laparoscopic abdominal surgery easier and safer for beginners in laparoscopic surgery and skilled surgeons in advanced laparoscopic surgery1. This simple technique of intracorporeal finger assistance permits a fast and safe solution in selected surgical cases ${ }^{2}$. In the hands of an experienced laparoscopic team, FALRP (Finger assisted laparoscopic resection of prostate) for large benign prostatic hyperplasia is a feasible, reproducible alternative approach to open surgery. It is safe, with minimal intraoperative haemorrhage and post-operative complications, and a shorter convalescence period than open surgery ${ }^{3}$. Finger assisted laparoscopic surgery can be performed in complicated surgery where distinguishing normal and inflamed tissues is must for safer surgery. This technique restores the tactile ability and allows gentle and safe blunt dissection by the operating surgeon ${ }^{4}$. This technique are used for various procedures like Totally Extra Peritoneal mesh repair for inguinal hernia ${ }^{5}$, radical prostatectomy, myomectomy ${ }^{6,7}$, to control bleeding, complicated appendectomy etc. Conversions were more frequent in the early part of learning curve, fewer among the group of experts in advanced laparoscopic surgery, conversions were due to an 
intraoperative complication and conversion was done because of technical difficulties ${ }^{8}$.

\section{Conclusion}

Even though, the number of cases involved in our study are only three, the FALS can be applicable in all patients with complex CBD stone ${ }^{14}$ with failed ERCP and LCBDE. Because, FALS procedure for CBD stone extraction reduces the operative time and surgical cost significantly. I order to say that this novel procedure can be adapted as a standard method we need to wait for large case series. Also, we need to do further more case study to know about its reproducibility and success rate in patients with failed ERCP and LCBDE in clearing the CBD stones.

\section{Conflicts of interest}

The authors declare that there no conflicts of interests regarding the publication of this paper.

\section{References}

[1] Ichihara T, Takada M, Fukumoto S, Yasuda T, Kuroda Y. A novel technique of finger-assisted laparoscopic surgery. Am J Surg. 2004 Feb;187(2):285-7.

[2] Tonietto G, Pintaldi S. Finger-assisted laparoscopic surgery. J Laparoendosc Surg. 1994 Jun; 4(3):205-7.

[3] Jean-Luc Hoepffner'Correspondence information about the author Jean-Luc HoepffnerEmail the author JeanLuc Hoepffner, Richard Gaston, Thierry Piechaud, Denis Rey, Camille Mugnier, BertinNjinou, Alberto Pansadoro, SasBarmoshe, Mata Emmanuel Lufuma. Finger Assisted Laparoscopic Retropubic Prostatectomy. European Urology Supplements Volume 5, Issue 19, November 2006, Pages 962-967

[4] Namir Katkhouda, MD (FACS)'Correspondence information about the author MD NamirKatkhouda, Rodney J Mason, MD, Eli Mavor, MD, Guilherme M.R Campos, Raymond T Rivera, MD, Michael B Hurwitz, $\mathrm{MD}$, Donald Waldrep, MD. Laparoscopic fingerassisted technique (fingeroscopy) for treatment of complicated appendicitis. Journal of the American College of Surgeons.

[5] R Kothari D Sharma U Somashekar. Finger-assisted totally extra-peritoneal laparoscopic hernia repair: A simple aid to unfolding the mesh for the novice.Asian $\mathrm{J}$ EndoscSurg 5(2012)152.

[6] YannisPrapasMD, PhDIoannisKalogiannidisMD, PhDNikosPrapasMD, PhD.Laparoscopy vs laparoscopically assisted myomectomy in the management of uterine myomas: a prospective study. American Journal of Obstetrics and Gynecology Volume 200, Issue 2, February 2009, Pages 144.e1144.e6.

[7] JiaWuZhong-FangZhangYe-LinXiePeng-ChenJiangLiPingChenRu-XiaShi. A novel modification of conventional laparoscopic myomectomy using manual assistance for multiple uterine myomas. European Journal of Obstetrics \& Gynecology and Reproductive Biology Volume 164, Issue 1, September 2012, Pages 74-78
[8] Priego P1, Lobo E, Sanjuanbenito A, Martínez Molina E, Pérez de Oteyza J, Ruiz Tovar J, Rodríguez Velasco G, Fresneda V. Causes of conversion in laparoscopic surgery for gastroesophageal reflux disease: an analysis of our experience. Rev EspEnferm Dig. 2008 May;100(5):263-7

[9] Ale'xis Sanchez, MD, Omaira Rodriguez, MD, Omar Bellorín, MD,RenataSa'nchez, MD, Gustavo Benítez, MD. Laparoscopic Common Bile Duct Exploration in Patients With Gallstones and Choledocholithiasis. JSLS. 2010 Apr-Jun; 14(2): 246-250.

[10] Arya N1, Nelles SE, Haber GB, Kim YI, Kortan PK. Electrohydraulic lithotripsy in 111 patients: a safe and effective therapy for difficult bile duct stones. Am J Gastroenterol. 2004 Dec;99(12):2330-4.

[11] Uma shanker, Finger Assisted Laparoscopic Surgery CBD https://www.youtube.com/watch?v=QOOjPFsNgs\&t=79s, Published on Sep 5, 2017, seen on 11 April; 2019.

[12] J Hochberger, J Bayer, A May, S Mühldorfer, J Maiss, E G Hahn, C Ell. Laser lithotripsy for difficult bile duct stone:results in 60 patients using rhodamine $6 \mathrm{G}$ dye laser with optical stone tissue detection system. Gut 1998;43:823-829.

[13] Swahn F, Edlund G, Enochsson L, Svensson C, Lindberg B, Arnelo U. Ten years of Swedish experience with intraductal electrohydraulic lithotripsy and laser lithotripsy for the treatment of difficult bile duct stones: an effective and safe option for octogenarians. SurgEndosc. 2010 May;24(5):1011-6. doi: 10.1007/s00464-009-0716-8. Epub 2009 Oct 23.

[14] Usküdar O1, Parlak E, Dişibeyaz S, Köksal AS, Çiçek B, Kılıç ZM, Odemiş B, Saşmaz N. Major predictors for difficult common bile duct stone. Turk J Gastroenterol. 2013;24(3):260-5. 